\title{
Transition prediction for oblique breakdown in supersonic boundary layers with uncertain disturbance spectrum
}

\author{
G. Serino, O. Marxen†, F. Pinna $\ddagger$ P. Rambaud ${ }^{\S}$ and T.E. Magin $₫$ \\ Aeronautics and Aerospace Department, von Karman Institute for Fluid Dynamics, Belgium
}

\begin{abstract}
Laminar to turbulent transition in supersonic boundary layer is numerically investigated by combining linear stability theory and Uncertainty Quantification. Linear stability theory is used to determine the $N$-factor for the $e^{N}$ transition prediction method for a Mach 6 flat plate test case. Transition onset location is determined by using the $N$-factor experimentally obtained in the facility where the test was carried out. Uncertainty quantification is used to compute the probability of transition within the intermittency region downstream of the transition onset. The stochastic approach allows to model the transition region as in the experimental cases since a gradual passage from the laminar to the turbulent flow is obtained. The probability of transition resembles the shape of the skin friction or the heat flux distribution generally observed in the experiments within the transition region. Here we focus on the transition zone rather than on the onset location.
\end{abstract}

\section{Nomenclature}

$F \quad$ Frequency of the oblique waves $(H z)$

$M_{\infty}$ Free stream Mach number

$N \quad$ Amplification factor for the $e^{N}$ transition prediction method

$R_{\infty} \quad$ Free stream Reynolds number

$T_{w} \quad$ Wall temperature $(K)$

$T_{\infty} \quad$ Free stream temperature $(K)$

$c d f$ Cumulative density function

$l_{s c} \quad$ Reference length $(m)$

$n_{s} \quad$ number of samples

$n_{p} \quad$ order of interpolating polynomials

$n$ spot propagation rate

$p_{\infty} \quad$ Free stream pressure $(\mathrm{Pa})$

$p d f$ Probability density function

\section{Greek Symbols}

$\alpha \quad$ Streamwise wave number

*PhD Candidate, Aeronautics and Aerospace Department, von Karman Institute for Fluid Dynamics, Chaussée de Waterloo 72, B-1640 Rhode-St-Genèse, Mechanical and Aerospace Engineering Department, Université de Liège, 1 Chemin des Chevreuils, B-4000 Liège, AIAA member, gennaro.serino@vki.ac.be.

${ }^{\dagger}$ Postdoctoral researcher, Aeronautics and Aerospace Department, von Karman Institute for Fluid Dynamics, Chaussée de Waterloo 72, B-1640 Rhode-St-Genèse, AIAA member, olaf.marxen@vki.ac.be.

¥Research engineer, Aeronautics and Aerospace Department, von Karman Institute for Fluid Dynamics, Chaussée de Waterloo 72, B-1640 Rhode-St-Genèse, AIAA member, fabio.pinna@vki.ac.be.

${ }^{\S}$ Associate Professor, Aeronautics and Aerospace Department, von Karman Institute for Fluid Dynamics, Chaussée de Waterloo 72, B-1640 Rhode-St-Genèse, AIAA member, patrick.rambaud@vki.ac.be.

`Assistant Professor, Aeronautics and Aerospace Department, von Karman Institute for Fluid Dynamics, Chaussée de Waterloo 72, B-1640 Rhode-St-Genèse, AIAA member, thierry.magin@vki.ac.be. 
Spanwise wave number

Intermittency factor

Reduced frequency $\omega=2 \pi F l_{s c} / U_{\infty}$

propagation angle $\left({ }^{\circ}\right)$

\section{Acronyms}

$C F D$ Computational fluid dynamics

$D N S$ Direct numerical simulations

$L S T \quad$ Linear stability theory

$O W B$ Oblique wave breakdown

$P S E \quad$ Parabolyzed stability equations

QoI Quantity of interest

$U Q$ Uncertainty quantification

\section{Introduction}

The knowledge of the location of laminar-turbulent transition is crucial for a large number of technical applications, for instance for laminar airfoils of wind-turbulent blades and for thermal-protection system of hypersonic re-entry vehicles. Early transition on a wind-turbine blade may strongly affect its performance, such as lift and drag, as well as noise production. For a hypersonic vehicle, an accurate prediction of the transition location may allow to precisely define the dimensions of the thermal protection system. The development of credible engineering models for the prediction of laminar-turbulent transition is therefore a critically important task.

Transition prediction relies on experiments and numerical simulations. The former have been used to build correlation and empirical criteria which have been successfully adopted in the past. However, experiments are expensive and they can not reproduce the free stream conditions experienced in flight. In fact, the Reynolds number, the Mach number and the temperature ratio at the wall are constrained by the limitations of the facility where the test is performed. On the other hand, computational fluid dynamics (CFD) allows to perform simulations for flight conditions, but a reliable transitional model is still lacking, especially at high Mach number.

The difficulty to predict transition lies in its uncertain nature and, therefore, we propose to numerically investigate the transition mechanism occurring in supersonic boundary layers by combining deterministic simulations, uncertainty quantification and comparison with experimental data. A probabilistic approach taking into account some of the uncertainties regarding the input conditions can be used to investigate the intrinsic randomness of transition. In fact, in wind tunnel tests as well as in flight and in the atmosphere, free stream conditions such as the Mach number, the temperature or the pressure are known with intervals of uncertainty making transition prediction even more difficult. Therefore, the uncertainty quantification is a sound strategy to investigate this "stochastic process" by including probability in deterministic simulations. Results of this new approach are presented in terms of map of the computational domain where transition is most likely to occur. In particular, the work is focus on transition caused by oblique wave breakdown which is the case most occurring in supersonic boundary layer.

\section{I.A. Oblique Wave Breakdown}

The laminar to turbulent transition scenario is characterized by three main stages: receptivity, linear amplification and non-linear breakdown. The receptivity deals with how the free stream disturbances are modified and converted into boundary layer waves. In the linear amplification stage, the amplitudes of the boundary layer waves are linearly amplified in space and time until they reach a certain percentage of the free stream value. Then, they start to grow non-linearly until breakdown to turbulence occurs.

In a supersonic boundary layer, the breakdown of oblique first mode waves is recognized as one of the dominant mechanism which causes transition. ${ }^{1}$ This transition scenario is characterized by the nonlinear interaction of two oblique waves with opposite wave angles which create a streamwise vortex. Transition 
is thus generated by the mutual interaction of the vortex and the oblique modes, which leads to a rapid breakdown to small scale turbulence. Generally, a very rapid breakdown is observed when the oblique waves reach an amplitude of about $4 \%$.

When transition is caused by a pair of oblique waves with opposite wave angles, it is defined as Oblique Wave Breakdown (OWB). A detailed review of this transition scenario in incompressible flows is given by Berlin $^{5}$ who also carried out several DNS computations which were compared to experimental data to better understand this mechanism. It was demonstrated that transition breakdown mainly consists of three stages: first a non-linear generation of a streamwise vortex by the oblique waves, second a transient growth of streaks from the vortex by the lift-up effect, and third a breakdown of the streaks due to secondary instability.

First studies of this transition scenario occurring in supersonic boundary layer were carried out by Chang and Malik ${ }^{6,}{ }^{7}$ Non linear PSE computations showed that oblique breakdown could be originated by initial disturbances lower than the classical secondary instability. They also observed a good agreement between LST and DNS computations in terms of amplitude of the disturbances until the point of non linear breakdown where perturbations start to grow non linearly. Finally, Meyer et al. ${ }^{9}$ used DNS to demonstrate that oblique breakdown can lead to a fully developed turbulent boundary layer and therefore it is a relevant mechanism for transition in two-dimensional supersonic boundary layers.

Linear Stability Theory (LST) ${ }^{2}$ assumes wave-like disturbances in the mean flow which can be modeled as a function of spatial coordinates $x, y, z$ and of time $t$. Here $x$ is the streamwise direction, $z$ the spanwise direction and $y$ the direction normal to the wall. LST is in general a good model to for describing the growth of oblique waves as demonstrated by Marxen ${ }^{8}$ through comparison with DNS.

$$
v(x, y, z, t)=\hat{v}(y) e^{i(k \alpha x+l \beta z-m \omega t)}, \quad \alpha=2 \pi / \lambda_{x} \quad \beta=2 \pi / \lambda_{z}
$$

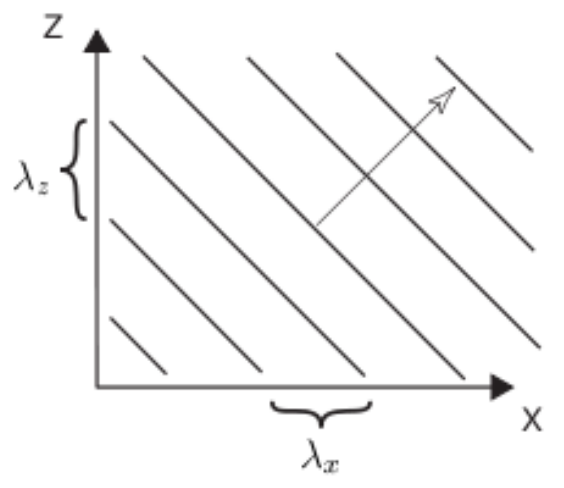

(a)

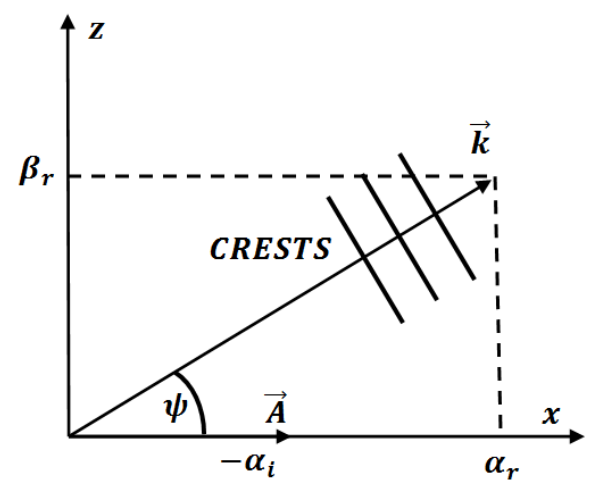

(b)

Figure 1. (a)Wave propagating in the mean flow with streamwise wave number $\lambda_{x}$ and spanwise wave number $\lambda_{z}$, (b) Notation for the oblique waves

Here, $\alpha$ and $\beta$ are the respectively the streamwise and spanwise wave number as represented in Fig. 1(a). The frequency $\omega$ is related to the streamwise wave number $\alpha$ by the phase speed $c, c=\omega / \alpha$, at which the disturbances travels downstream. In general, $\alpha, \beta$ and $\omega$ are complex. In the current work, we consider two dimensional boundary layer, hence $\beta$ is a real number. Moreover, the spatial approach for linear stability theory is used thus $\omega$ is a real number while $\alpha$ is complex. In Fig. 1(b), $\vec{k}$ represents the envelope direction of the crests of the waves traveling in the domain while $\vec{A}$ represents their most amplified direction. In the present work, the vector $\vec{A}$ is always aligned with the $x$ axis since only two dimensional base flows are analyzed. In fact, in the LST computations, it is necessary to compute the angle $\psi=\tan ^{-1}\left(\beta_{r} / \alpha_{r}\right)$ in order to properly represent the oblique waves. The $\psi$ angle requires to compute both $\alpha_{r}$ and $\beta_{r}$ in an interactive way since they are not independent. Arnal ${ }^{3}$ and Ferrier ${ }^{4}$ describe different procedures for the computation of the propagation angle and, in addiction, for the evaluation of the imaginary part $\beta_{i}$ of the spanwise wave number. Here we assume $\beta_{i}=0$. 


\section{I.B. Linear stability theory}

Transition is predicted via the $e^{N}$ method which applies linear stability theory. A detailed overview of this method is given by Arnal. ${ }^{3}$ When the spatial approach is used, the integration of the amplification rates $\alpha_{i}$ along the streamwise direction gives the $N$ factor as follows:

$$
N=\log \frac{A}{A_{o}}=\int_{x_{0}}^{x}-\alpha_{i} d x
$$

In Eq. $2, A_{0}$ is the initial amplitude of the disturbances at the location $x_{0}$ where they start to be amplified. According to the $e^{N}$ method, transition occurs when the computed $N$ factor equals the value determined at the experimental transition location for a specific wind tunnel. In general, for subsonic facilities, the $N$ factor for transition is around $8 \div 9$ while it is $4 \div 5$ at higher Mach number as in hypersonic conditions. Examples of application of the method are the experiments carried out by Horvath ${ }^{10}$ in the NASA Langley Mach 6 wind tunnel where transition onset is at $N$ equal to 3.8. The $N$-factor depends on the turbulence level of the free stream and therefore it can vary from $4 \div 5$ in noisy facilities up to $12 \div 13$ in flight conditions. The limiting $N$ - factor is not known in advance and must be determined by calibration to wind tunnel or flight tests, hence the $e^{N}$ approach is considered as a semi-empirical method for transition prediction.

\section{I.C. Uncertainty Quantification}

Iaccarino et al. ${ }^{12}$ define uncertainty quantification (UQ) as a probabilistic approach to determine confidence levels on the results of numerical simulations. A real physical system is characterized by intrinsic uncertainties related to the input conditions and UQ aims at studying how they affect the numerical results focusing on some quantities of interest (QoI). The UQ strategy consists in defining the input uncertainties, in studying how they propagate in the simulations and finally in defining the margins and error bars on the QoI.

Pecnik et al. ${ }^{13}$ have studied the effects of input uncertainties on transition prediction of transonic gas turbine compressors. Numerical results are compared with experimental data obtained at the von Karman Insitute for Fluid Dynamics for several turbine guide vane test cases. Uncertainties are classified into aleatory and epistemic both due to lack of knowledge, the former on the input conditions while the latter on the RANS transitional model parameters. It is shown that, when uncertainties are taken into account, results are closer to the experiments with respect to the fully deterministic simulations. Applications of the UQ strategy to high speed flows are presented by Iaccarino ${ }^{14}$ for two classical problems in unsteady compressible fluid dynamics: the Riemann problem and the Woodward-Colella forward step flow. The propagation of the uncertainties on the solutions is derived with both non-intrusive and intrusive techniques. A non-intrusive technique consists in several repetitions of the original deterministic models. Examples are the Monte Carlo method or the stochastic collocation approaches. On the other hand, an intrusive technique in based on the modification of the original deterministic models as in the polynomial chaos approach where a polynomial expansion is used to model a certain variable or parameter. Non-intrusive technique are usually slower to converge with respect to the intrusive approaches when the number of uncertain parameters to be analyzed increases. This is a well known issue in uncertainty quantification which is addressed to as the curse of dimensionality.

\section{Methodology}

All the stages of the transition scenario described by the linear stability theory have to be accurately modeled in numerical simulations. In this work, only the results of the receptivity process is modeled by considering a certain probabilistic distribution for the boundary layer waves in terms of their frequency $(F)$ and propagation angle $(\psi)$. This is justified since we do not know much about the free stream spectrum. Therefore, rather than assuming a probability distribution for the free stream spectrum and applying a receptivity model, we may as well model the $p d f$ of the boundary layer spectrum in the first place. The nonlinear breakdown is modeled by considering a breakdown criterion based on the $N$-factor at the experimental transition onset. In oblique breakdown, transition is very quick and guaranteed to take place once an amplitude of the order of $1 \%$ has been reached. The linear amplification phase is solved through a stability analysis on boundary layer profiles derived by Blasius's self similar solution with the stability code developed by Pinna. ${ }^{15}$ 
A test case is analyzed and compared with experiments carried out by Dilley ${ }^{16}$ and Horvath. ${ }^{10}$ The free stream conditions are indicated in Tab. 1 with the adiabatic wall condition applied to the wall.

Table 1. Test case and free stream conditions

\begin{tabular}{cccccc} 
Test case & $M_{\infty}$ & $T_{\infty}$ & $p_{\infty}$ & $R e_{\infty}$ & $T_{w}$ \\
\hline Flat Plate & 6.0 & $65.4 \mathrm{~K}$ & 0.022 bar & $26.4 \times 10^{6} 1 / \mathrm{m}$ & $106 \mathrm{~K}$
\end{tabular}

Linear stability theory has been implemented in a numerical code to investigate stability properties of boundary layer profiles. The stability code VESTA developed by Pinna ${ }^{15}$ in his PhD thesis is used in this work. The toolkit is executable in MATLAB environment and it allows for the computation of the stability properties with the spatial approach for 2-D velocity profiles both for incompressible and compressible flows. The velocity profiles can be computed through the self-similar solution of the boundary layer equations or they can also be obtained by means of CFD computations. The integration of the amplification rate to compute the $N$-factor or the neutral stability curves are just two of the possible outputs of the code.

The general $U Q$ strategy can be summarized as follows :

1. Definition of the uncertainties: the dimensional frequency $(F)$ and the direction of the crests of the waves $(\psi)$ have been selected as input uncertainties. A normal distribution has been chosen to describe them with the values indicated in Tab. 2. Frequencies have been varied within $50 \div 250 \mathrm{kHz}$ as these are the typical values related to the first mode in hypersonic boundary layers. On the other hand, the propagation direction of the waves has been varied within $40^{\circ} \div 70^{\circ}$ corresponding to the first mode of Mack. ${ }^{3}$

Table 2. Input uncertainties

\begin{tabular}{cccc} 
Parameter & $\mu$ & $\sigma$ & Range \\
\hline Frequency $(F)$ & $200 \mathrm{kHz}$ & $20 \mathrm{kHz}$ & $50 \div 250 \mathrm{kHz}$ \\
Amplification angle $(\psi))$ & $60^{\circ}$ & $2^{\circ}$ & $40^{\circ} \div 70^{\circ}$
\end{tabular}

2. Propagation: a transfer function, which relates the two uncertain parameters to the amplification ratio $N=N($ Frequency, $\psi)$, and a sampling method allows us to propagate the uncertainties through the numerical simulations. Transfer functions directly relate the input to a quantity of interest and, in the current work, they are provided by the linear stability analysis. In fact, for each combination of the input uncertain parameters, the $N$ factor has been obtained on the computational domain. The transfer function has been discretized using a stochastic collocation grid where the collocation points are given by the roots of the Legendre polynomial. The transfer function is then used to evaluate the QoI for each samples on the physical intervals. The Monte Carlo technique has been chosen in the current work as it is a non-intrusive technique based on several independent solutions (repetitions) of the original system. The two-dimensional interpolation has been then applied by using the Lagrange technique in which the two-dimensional multipliers are obtained as a tensor product of the monodimensional multiplier. The order of the interpolating polynomial depends on the number of grid points of the stochastic collocation space and it can be varied until convergence is achieved. Thus, the amplification factor $N$ is computed for each random samples and the empirical $p d f_{N}$ is obtained for each $\mathrm{x}$-station of the computational domain.

3. Output: the probability distribution of the $N$-factor for each station of the computational domain is the output of the analysis. Since the work aims at modeling the transition region, results are presented in terms of probability to have transition at a fixed location of the domain. Thus, the probability gradually rises from 0 (laminar flow) at the transition onset up to 1 (turbulent flow) within the transition region. The probability is computed as the $c d f$ of the $p d f$ of the $N$-factor integrated up to the $N$ value at the experimental transition onset. 


\section{Results}

\section{III.A. Stability analysis and uncertainty quantification}

First linear stability analysis has been performed to obtain the transfer function to relate the input uncertainties to the $N$-factor. An example of the transfer function is represented in Fig. 2(a) for the x-station corresponding to $R e_{x}=12 \times 10^{6}$. The transfer function has a non linear behavior and it has to be properly described by choosing the collocation points on the stochastic grid. In addition, in Fig. 2(b) the N-factor is represented as a function of the frequency $(F)$ and of the $R e_{x}$ along the computational domain for a fixed amplification angle $\psi=60^{\circ}$.

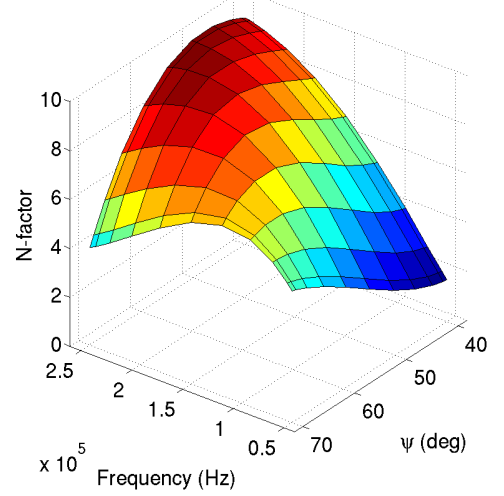

(a) Transfer function $N=N(F, \psi)$ : $\operatorname{Re}_{x}=12 \times 10^{6}$

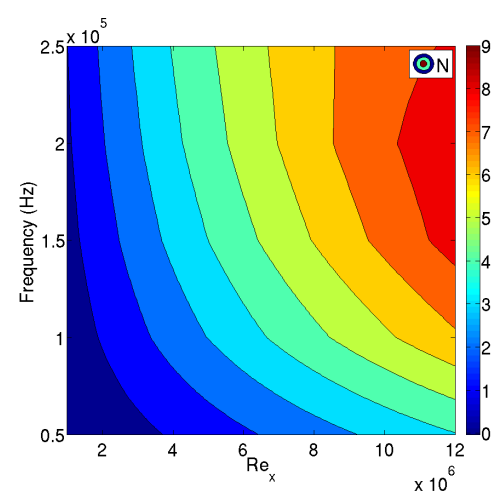

(b) N-factor as function of $R e_{x}$ and $F$ : $\psi=60^{\circ}$

Figure 2. Transfer function obtained with linear stability theory

Then, the uncertainty quantification approach has been applied using the probability density functions for the uncertain parameters which are represented in Fig. 3.

A Monte Carlo sampling is used due to its simplicity and it is particularly good for cases with many uncertain parameters since it does not suffer from the "curse of dimensionality". In Fig. 3, for illustration purpose, few random samples are represented on the physical space together with the collocation points on the tensor grid obtained with the quadrature rule.
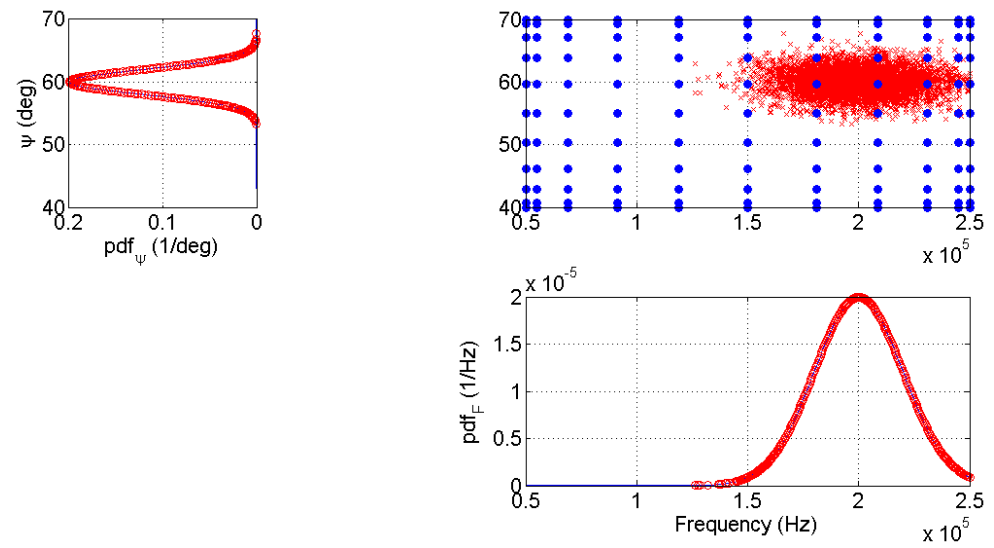

Figure 3. Sampling of the input pdf : samples (red crosses), collocation points (blue circles)

Finally, in Fig. 4, the output probability density function $\left(p d f_{N}\right)$ is represented for illustration purpose on a reference station. The corresponding cumulative distribution function, $c d f_{N}$, is then used to determine the probability to have an amplification factor greater than the threshold value related to the transition onset. 


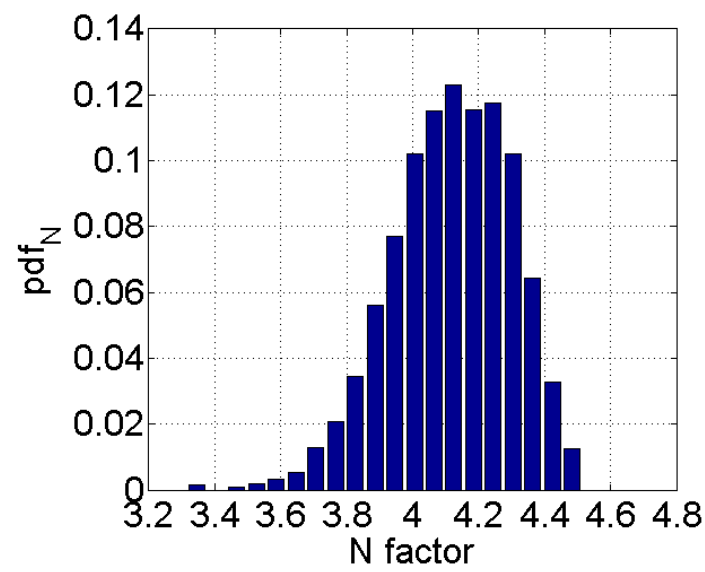

Figure 4. Amplification factor probability density function : $R e_{x} \approx 4 \times 10^{6}$ )

\section{III.B. Convergence analysis}

A convergence study has been carried out by varying the order of the interpolating polynomials for the input parameters and the number of samples. The mean and the variance of the output $p d f$ for the $N-$ factor are considered to evaluate the error and to define the convergence. In order to properly describe the output $p d f$ for $N$ - factor, the number of bins has been varied. The principle behind histograms is that the area of each bar represents the fraction of a probability distribution within each bin. In the current work, 20 bins are chosen to describe the output $p d f$ and to compute the probability of transition. The convergence is then assumed to be reached when the percentage variation of both the mean and the variance of the output $p d f$ of the $N$ - factor is below 1\%. At a reference station, the experimental transition onset location has been considered to observe the variation of the distribution of the amplification rate. The errors are defined as follows :

$$
\operatorname{err}_{\mu_{i}}=\frac{\left(\mu_{i}-\mu_{r e f}\right)}{\mu_{r e f}}, \quad \operatorname{err}_{\sigma}=\frac{\left(\sigma_{i}-\sigma_{r e f}\right)}{\sigma_{r e f}}
$$

In Eq. 3, the error at the $i$ variation of the number of the samples or of the order of the polynomials, is computed as the difference of the mean $(\mu)$ or the variance $(\sigma)$ respect to the true value. This is assumed to be obtained with the largest number of samples $\left(n_{s}=15000\right)$ and with the higher polynomial order $\left(n_{p}=17\right)$ respectively for the first and the second estimation of the error.

First, the number of samples has been progressively increased until convergence of the mean and of the variance of the output $p d f$ has been achieved. The Monte Carlo sampling technique allows to reduce the percentage error by a proportionality factor of $1 / \sqrt{n_{s}}$ being $n_{s}$ the number of samples. The percentage error of the mean and the variance of the output $p d f_{N}$ is reported in Tab. 3 and compared to the theoretical error in Fig. 5(a). It can be noticed that the percentage error on the variance is always above that for the mean. When the number of samples is 10000 , the error on the mean is reduced to $0.03 \%$ and it is $0.80 \%$ on the variance of the output $p d f_{N}$. Therefore, it is possible to use a number of samples equal to 15000 to have convergence on the output $p d f$.

\begin{tabular}{c|c|c|c|c|c|c|c}
$n_{s}$ & 10 & 50 & 100 & 500 & 1000 & 5000 & 10000 \\
\hline err $_{\mu_{N}}$ & 0.0257 & 0.0079 & 0.0010 & 0.0015 & 0.0005 & 0.0013 & 0.0003 \\
\hline err $_{\sigma_{N}}$ & 0.2215 & 0.0240 & 0.1057 & 0.0192 & 0.0384 & 0.0021 & 0.0080
\end{tabular}

Table 3. Relative error $\left(R e_{x}=3.5 \times 10^{6}\right)$ : variation of the samples

Then, with the number of samples which allows convergence (i.e. $n_{s}=15000$ ), the order of the interpolating polynomials on the frequency and on the amplification angle has been varied. The order of the polynomials $\left(n_{p}\right)$ is equal to the number of collocation points used for the frequency and the amplification 
angle to build the tensor grid on which the samples are then projected. The percentage error of the mean and the variance of the output $p d f_{N}$ is reported in Tab. 4 and represented in Fig. 5(b). Convergence is achieved with a $n_{p}=17$ degree interpolating polynomial which allows to have a residual error below $1 \%$ for both the mean and the variance of the output distribution of the $N$-factor. Therefore, for the current case we used $n_{p}=17$ to achieve convergence.

\begin{tabular}{c|c|c|c|c|c|c|c}
$n_{p}$ & 3 & 5 & 7 & 9 & 11 & 13 & 15 \\
\hline $\operatorname{err}_{\mu_{N}}$ & 0.0289 & 0.0335 & 0.0089 & 0.0005 & 0.0026 & 0.0010 & 0.0020 \\
\hline $\operatorname{err}_{\sigma_{N}}$ & 0.1060 & 0.1730 & 0.0996 & 0.0186 & 0.0478 & 0.0175 & 0.0064
\end{tabular}

Table 4. Relative error $\left(R e_{x}=3.5 \times 10^{6}\right)$ : variation of the order of the polynomials

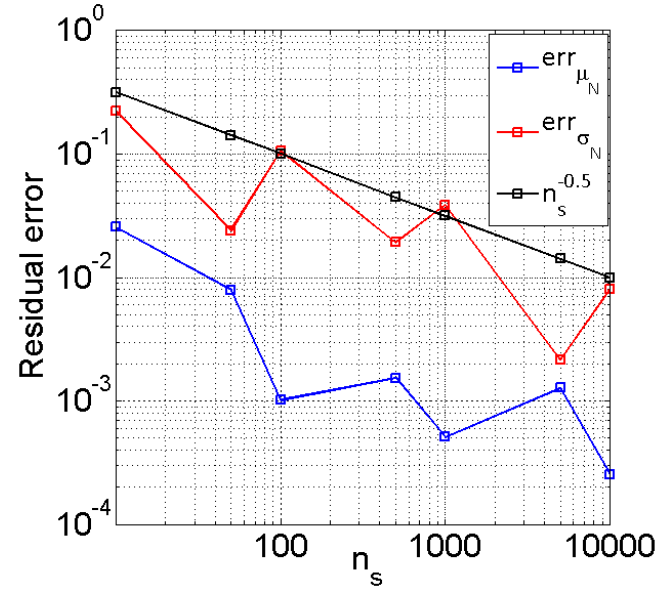

(a) variation of the samples

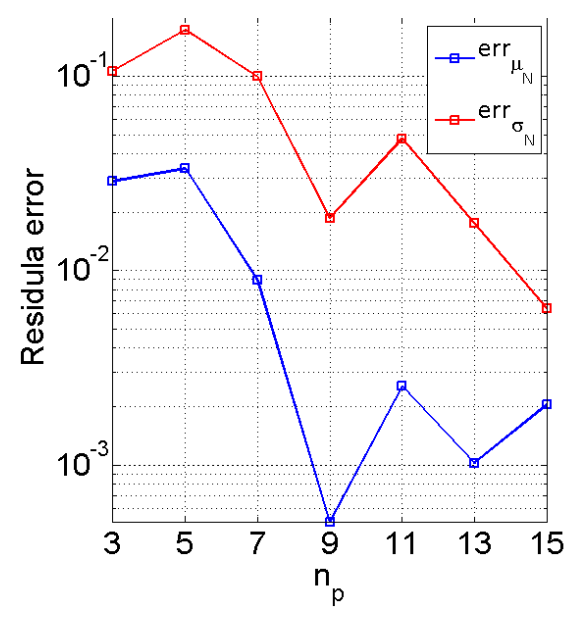

(b) variation of the order of the polynomials

Figure 5. Percentage error of the output pdf on its mean and variance $\left(\operatorname{Re}_{x}=3.5 \times 10^{6}\right)$

\section{III.B.1. Comparison with experiments}

The cumulative distribution function has been used to compute the probability to have an amplification ratio which exceeds the value determined at the experimental transition onset. An amplification ratio equal to $N=3.8$ is used as it is indicated by Dilley ${ }^{16}$ for the flat plate test case. In Fig. 6 this probability is represented on the flat plate as a function of the reference Reynolds number $R e_{x}=U_{\infty} x / \nu_{\infty}$ and compared with the experimental data and CFD computations. Experiments have been carried out in the 20 inches Mach 6 wind tunnel at NASA Langley Research Center which is a conventional disturbances wind tunnel whose $N$-factor for transition is varying between $3.8 \div 4.5$ respectively for cold and adiabatic wall conditions as indicated by Horvath. ${ }^{10}$ Transition onset is obtained with the tangent cone method from the heat flux distribution represented as the non-dimensional Stanton number $(S t)$. Experiments and CFD computations show that transition onset occurs at $R e_{x} \approx 3.5 \times 10^{6}$ where the experimental Stanton number starts to grow. The computed probability of transition rises from 0 (i.e. laminar flow) to 1 (i.e. turbulent flow) with an S-shape resembling the rising of the experimental heat flux in the transition region.

In Fig. 7(b), the effect of the variation of the variance $\sigma_{F}$ of the input $p d f$ for the frequency (Fig. 7(a)) is represented. An increasing of the variance leads to a parallel increasing of the transition length since the probability is distributed on a wider range of frequencies in the considered interval. The variation of the variance can be therefore considered a way to match the experimental data in terms of transition onset and transition length. It is important to say that a normal distribution is generally characterized also by negative values. Here the $p d f$ has been limited to the intervals defined in Tab. 2 thus avoiding non physical values, such as negative frequencies or propagation angles, for both the uncertain parameters.

For long times and hence very many realizations, the probability represented in Fig. 6 could be interpreted as the intermittency $\gamma$ which represents the turbulent/laminar time ratio. In Fig. 8 the computed probability 


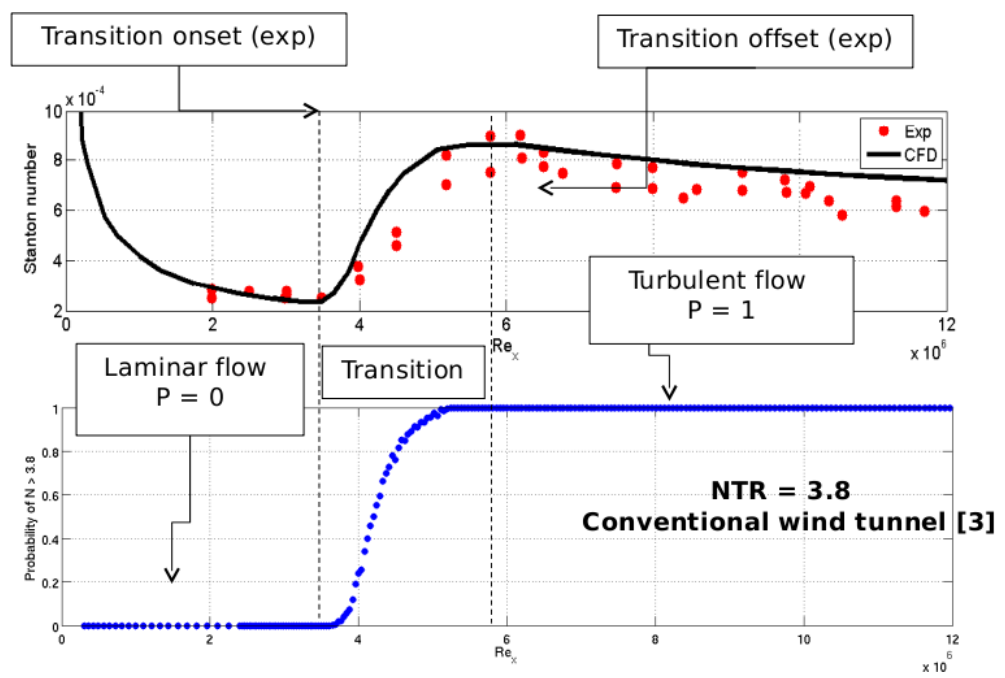

Figure 6. Comparison between numerical ${ }^{16}$ (black line), experimental ${ }^{16}$ (red dots) Stanton number distribution and the probability of transition (blue dots) on the computational domain for Test Case I

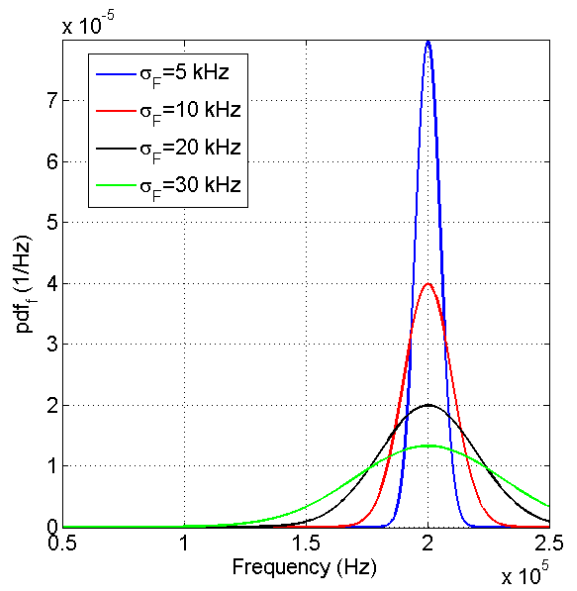

(a) pdf of the Frequency

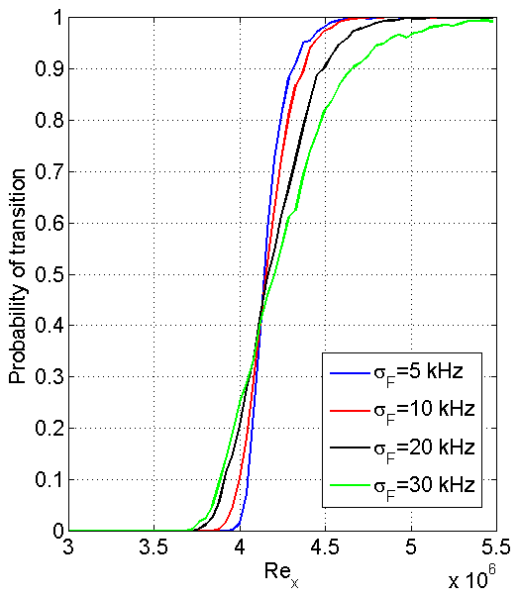

(b) probability of transition

Figure 7. Effect of the variation of the variance of the input pdf for the Frequency on the transition region 
is compared to Narasimha's intermittency model ${ }^{20}$ defined as follows :

$$
\gamma(x)=1-\exp \left[-\left(x-x_{t}\right)^{2} \frac{n \sigma}{U}\right]=1-\exp \left[-A\left(\frac{x-x_{t}}{\lambda}\right)^{2}\right]
$$

Eq. 4 models the distribution of the intermittency in the transition region. Thus, the equation is valid right after the transition onset $\left(x>x_{t}\right)$. Here, $n$ is the spot formation rate per unit time and per unit distance in the spanwise direction, while $\sigma$ is the spot propagation parameter which is $\approx 0.25$ according to the experimental observations of Narasimha in $1978 .^{20}$ The equation can be also reduced to the form presented on the right hand side where $A=0.412$ is a constant and $\lambda=\left.x\right|_{\gamma=0.75}-\left.x\right|_{\gamma=0.25}$ is a parameter related to the transition length. This parameter has been obtained from the experimental heat flux distribution presented in Horvath. ${ }^{10}$ The Stanton number has been normalized respect to the laminar and the fully turbulent value. Therefore, a curve going from 0 (laminar flow) to 1(turbulent flow) has been obtained in the transition region. Assuming that this curve actually represents the measured intermittency in the transition region, the $x$ coordinates where $\gamma=0.25$ and $\gamma=0.75$ have been retrieved and plugged in Eq. 4. For the assumptions made on the uncertain parameters, the computed probability fits the model in Eq. 4. Therefore, the approach presented here could be used to retrieve the intermittency distribution in the transition region to be applied in a transitional model.

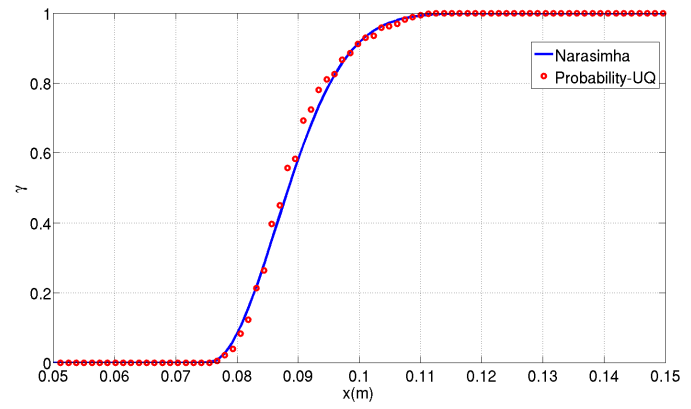

Figure 8. Comparison between the computed probability and Narasimha's intermittency

\section{Conclusion}

The $e^{N}$ method has been coupled to the uncertainty quantification approach in order to take into account probability in transition prediction. A probability distribution has been assumed for both the frequency and the propagation angle of the oblique waves. The strategy has been applied to a test case in which transition was experimentally observed. We assumed here that transition was caused by the non-linear breakdown of the oblique waves. The $N$-factor was chosen such that the transition onset matched the experimental observation. The probabilistic approach allows to obtain a probability which could be interpreted as the intermittency factor when the number of realizations (samples) is sufficiently large.

The present work demonstrated that the integration of deterministic theory, namely LST, and probabilistic approach (UQ) is a promising strategy in laminar to turbulent transition studies for hypersonic applications. The advantage of the approach is due to the possibility to predict the transition length, assuming that we know the $N$-factor corresponding to the experimental transition onset. The limitation is that, as in $e^{N}$ transition method, the $N$-factor for transition has to be known a priori through previous experiments and LST computations. In addition, it is possible to extend the approach to investigate the impact of other sources of uncertainties on the transition location. Although the effect of the frequency and the propagation angle has been presented in the paper to study the Oblique Wave Breakdown mechanism, the free stream Mach number or the wall temperature can be varied and combined to quantify the impact on the transition onset.

Finally, the obtained result give precious indications on how to model the transition region since a S-shaped probability was obtained. This means that, when uncertainties are taken into account, transition is not an abrupt switch from the laminar to the turbulent flow but it is a gradual passage which better represents what happens in reality. This S-shape can be used to model the transition region in CFD simulations by 
combining the computed probability/intermittency to the laminar and turbulent solution. In particular, this last part will be presented in future works by the authors.

\section{Acknowledgment}

The research of G. Serino is sponsored by the "Founds de la Recherche dans l'Industrie et l'Agriculture" (FRIA), the research of O. Marxen and T.E. Magin by the "European Research Council Starting Grant" number 259354.

\section{References}

${ }^{1}$ Chau-Lyan Chang and Mujeeb R. Malik, Oblique-mode breakdown and secondary instability in supersonic boundary layers,Journal of Fluid Mechanics, vol. 273, pp 323-360, 1994

${ }^{2}$ Schlichting, H., Boundary Layer Theory, Springer, 1965.

${ }^{3}$ Arnal D.,Special Course on Progress in Transition Modeling, AGARD report 793, 1993.

${ }^{4}$ Ferrier M., Analyse de la stabilité et prévision de la transition laminaire/turbulent de lécoulement proche paroi sur l'avantcorps d'un véhicule hypersonique, PhD Thesis in Fluid Mechanics, Université d' Orléans, 2008.

${ }^{5}$ Berlin, S., Oblique Waves in Boundary Layer Transition, Doctoral Thesis, Stockholm, 1998.

${ }^{6}$ Chang, C.L. \& Malik, M. R. Oblique-mode breakdown and secondary instability in a supersonic boundary layers using nonlinear PSE, Instability, Transition and Turbulence, Hussaini, M. Y., Kumar, A. \& Streett, C. L., editors, pp. $231-241$. Springer-Verlag, 1992.

${ }^{7}$ Chang, C.-L. \& Malik, M. R. Oblique-mode breakdown and secondary instability in supersonic boundary layer, J. Fluid Mech. 273, pp. 323-360, 1994.

${ }^{8}$ Marxen O., Iaccarino G. \& Shaqfeh E., Boundary-layer transition via spatially growing oblique waves, under consideration for publication in Journal of Fluid Mechanics, 2011.

${ }^{9}$ Mayer C., Von Terzi D. \& Fasel H., Direct numerical simulation of complete transition to turbulence via oblique breakdown at Mach 3, J. Fluid Mech., 2011.

${ }^{10}$ Horvath T.J., Berry S.A., Hollis B.R., Chang C.L. and Singer B.A., Boundary layer transition on slender cones in conventional and low disturbance Mach 6 wind tunnels, NASA Langley Research Center, AIAA 2002-2743.

${ }^{11}$ Robarge T.W. and Schneidert S.P., Laminar Boundary-Layer Instabilities on Hypersonic Cones: Computations for Benchmark Experiments, AIAA Paper 2005-5024, 35th Fluid Dynamics Conference, Toronto, June 2005.

${ }^{12}$ Iaccarino G., Eldred M., Doostan A., Ghattas O., Introduction to Uncertainty Quantification,SIAM CSE Conference, Miami, FL, 2009.

${ }^{13}$ Pecnik R., Witteveen J. \& Iaccarino G., Uncertainty quantification for laminar-turbulent transition prediction in RANS turbomachinery applications, 49th AIAA Aerospace Sciences Meeting including the New Horizons Forum and Aerospace Exposition, Orlando, Florida, AIAA 2011-660.

${ }^{14}$ Iaccarino G., Pettersson P., Nordstr J. \& Witteveen J., Numerical methods for uncertainty propagation in high speed flows, V European Conference on Computational Fluid Dynamics, ECCOMAS CFD 2011, Lisbon, Portugal, 14-17 June 2010.

${ }^{15}$ Pinna F., Numerical study of stability of flows from low to high Mach number, PhD Thesis, von Karman Institute for Fluid Dynamics and Universitá di Roma 'La Sapienza', 2012.

${ }^{16}$ Dilley A.D., Evaluation of CFD turbulent heating prediction techniques and comparison wit hypersonic experimental data, NASA/CR-2001-210837.

${ }^{17}$ Tumin A., Wang X. and Zhong X., Direct numerical simulation and the theory of receptivity in a hypersonic boundary layer, Physics of fluids, vol. 19, 2007.

${ }^{18}$ Beckwith E., Creel T., Chen Fang-Jenq, Kendall J., Free-Stream Noise and Transition Measurements on a Cone in a Mach 3.5 Pilot Low-Disturbance Tunnel, NASA Technical Paper 2180, September 1983.

${ }^{19}$ Malik M. R., Instability and transition in supersonic boundary layers, Energy Sources Technology Conference, New Orleans, LA, February 1984.

${ }^{20}$ Narasimha R., On the distribution of intermittency in the transition region of a boundary layer, Journal of Aeronautical Science, vol. 24, pp. 711-712, 1957. 